\title{
Are Crossed Actions of Reticulospinal and Vestibulospinal Neurons on Feline Motoneurons Mediated by the Same or Separate Commissural Neurons?
}

\author{
Piotr Krutki, ${ }^{1}$ Elzbieta Jankowska, ${ }^{1}$ and Stephen A. Edgley ${ }^{2}$ \\ ${ }^{1}$ Department of Physiology, Göteborg University, 40530 Göteborg, Sweden, and ${ }^{2}$ Department of Anatomy, University of Cambridge, Cambridge, CB2 3DY, \\ United Kingdom
}

\begin{abstract}
Both reticulo- and vestibulospinal neurons coordinate the activity of ipsilateral and contralateral limb muscles. The aim of this study was to investigate whether their actions on contralateral motoneurons are mediated via common interneurons. Two series of experiments were made on deeply anesthetized cats. First, the effects of stimuli applied within the lateral vestibular nucleus and to reticulospinal tract fibers within or close to the medial longitudinal fascicle in the medulla were tested on midlumbar commissural interneurons that projected to contralateral motor nuclei. EPSPs of vestibular origin were found in 16 of 20 (80\%) of the interneurons, all of which were excited monosynaptically by reticulospinal fibers. These EPSPs were evoked either monosynaptically or disynaptically. Second, the effects of stimuli applied at the same two locations were tested on contralateral motoneurons, selecting motoneurons in which large disynaptic EPSPs or IPSPs were evoked by reticulospinal fibers. When stimuli that were too weak to evoke any PSPs by themselves were applied together, similar EPSPs or IPSPs were evoked in all 26 motoneurons that were tested, indicating that spatial facilitation occurred premotoneuronally. Facilitation was strongest at those intervals optimal for summation of monosynaptic and/or disynaptic EPSPs evoked in commissural neurons by the earliest reticulospinal and vestibulospinal volleys. The same interneurons thus may be used by reticulospinal and vestibulospinal neurons to influence the activity of contralateral hindlimb muscles. Separate modulation of commands from these two descending neuronal systems may occur at the level of the interneurons that mediate disynaptic excitation of commissural neurons by reticulospinal and vestibulospinal neurons, thereby increasing their flexibility.
\end{abstract}

Key words: spinal cord; postural reactions; reticulospinal neurons; vestibulospinal neurons; commissural neurons; motoneurons

\begin{abstract}
Introduction
Two bulbospinal neuronal populations, vestibulo- and reticulospinal, coordinate movements of the left and right limbs. Direct monosynaptic excitation of motoneurons is quite specific because it involves mainly ipsilateral extensor motoneurons (by vestibulospinal neurons) or ipsilateral flexor motoneurons (by reticulospinal neurons) (Lund and Pompeiano, 1965; Grillner and Lund, 1968; Grillner et al., 1968, 1970, 1971; Lund and Pompeiano, 1968; Shapovalov, 1969; Wilson and Yoshida, 1969; Gossard et al., 1996) (however, see Floeter et al., 1993). The indirect actions of the vestibulo- and reticulospinal tract fibers are more widespread in flexor as well as extensor motoneurons both ipsilaterally and contralaterally, and similar actions often are evoked by these fibers in the same motor nuclei (Shapovalov, 1969; Grillner et al., 1970, 1971; Aoyama et al., 1971; Hongo et al., 1971; Maeda et al., 1975; Gossard et al., 1996; Jankowska et al., 2003).
\end{abstract}

\footnotetext{
Received March 21, 2003; revised June 26, 2003; accepted July 16, 2003.

This study was supported by National Institutes of Health Grant NS 40 863. We thank Rauni Larsson for her invaluable assistance during the experiments and for histological verifications.

Correspondence should be addressed to E. Jankowska, Department of Physiology, Göteborg University, Medicinaregatan 11, Box 432, 40530 Göteborg, Sweden. E-mail: Elzbieta.Jankowska@physiol.gu.se.

P. Krutki's present address: Department of Neurobiology, University School of Physical Education, 60-352 Poznal, Poland.

Copyright $\odot 2003$ Society for Neuroscience $\quad 0270-6474 / 03 / 238041-10 \$ 15.00 / 0$
}

The interneuronal pathways activated by vestibulo- and reticulospinal neurons thus may act jointly rather than independently.

Coordination between the actions of vestibular and reticular neurons would be easiest to achieve if they were mediated by the same spinal interneurons. However, there is evidence both for and against this possibility. In several studies both long propriospinal neurons (Alstermark et al., 1987a-c) and lumbar interneurons coexcited by reticulospinal and vestibulospinal fibers have been found (Kozhanov and Shapovalov, 1977; Skinner and Remmel, 1978; Davies and Edgley, 1994) that could mediate joint reticulo- and vestibulospinal actions. This was particularly true for interneurons of Davies and Edgley (1994), because these were last-order interneurons in pathways to ipsilateral hindlimb motoneurons. Interneurons coexcited by reticulospinal and vestibulospinal fibers also have been found in lamprey (Rovainen, 1979).

However, analysis of inhibitory pathways from vestibulo- and reticulospinal neurons indicated that disynaptic inhibition evoked in ipsilateral hindlimb motoneurons is mediated by distinct populations of interneurons. For instance, vestibulospinal fibers evoke disynaptic inhibition via interneurons responsible for Ia reciprocal inhibition from extensors to flexors (Grillner and Hongo, 1972; Hultborn and Udo, 1972) and for inhibition associated with crossed extensor reflexes (Bruggencate et al., 
1969), but not via interneurons mediating Ia/Ib nonreciprocal inhibition. In contrast, disynaptic inhibition evoked from the reticular formation has been shown to be mediated by interneurons in pathways of $\mathrm{Ia} / \mathrm{Ib}$ nonreciprocal inhibition and of flexor reflexes, but not of Ia reciprocal inhibition (Takakusaki et al., 2001). A study of disynaptic excitatory pathways to ipsilateral limb motoneurons similarly led to the conclusion that the vestibulo- and reticulospinal neurons operate via mainly separate populations of interneurons (Gossard et al., 1996).

The present study of disynaptic actions of vestibulo- and reticulospinal neurons on contralateral motoneurons demonstrates their mediation by the same interneurons. Our results thus lead to the conclusion that the activity of contralateral muscles may depend greatly on interactions of vestibulo- and reticulospinal tract fibers on commissural interneurons.

\section{Materials and Methods}

Preparation. Experiments were performed on six deeply anesthetized cats $(2.3-2.9 \mathrm{~kg})$. Anesthesia was induced by sodium pentobarbital $(40 \mathrm{mg} /$ $\mathrm{kg}$, i.p.) and maintained with $\alpha$-chloralose (in doses of $5 \mathrm{mg} / \mathrm{kg}$, i.v., up to a total of $40-50 \mathrm{mg} / \mathrm{kg}$ ). Full anesthesia was verified from the lack of withdrawal and corneal reflexes before neuromuscular transmission was blocked and by monitoring of the diameters of the pupils, heart rate, and blood pressure throughout the experiment. Additional doses of $\alpha$-chloralose were given if the pupils dilated or if the blood pressure or heart rate rose in response to stimulation. Mean blood pressure was kept between 90 and $130 \mathrm{mmHg}$, and the $\mathrm{CO}_{2}$ level in the expired air was kept near $4 \%$ by adjusting the volume of artificial respiration and a rate of a continuous infusion of a bicarbonate buffer solution with $5 \%$ glucose (1-2 $\mathrm{ml} / \mathrm{kg}$ per hr). The animal's core temperature was maintained at $37-38^{\circ} \mathrm{C}$ with heating lamps. Bilateral pneumothorax was made to reduce respiratory movements and improve the recording stability. All of the experimental procedures were approved by Göteborg Ethics Committee and followed National Institutes of Health and European Union guidelines of animal care. At the end of the experiment an overdose of anesthetic was given until cardiac arrest.

A number of hindlimb nerves were dissected, transected, and mounted on stimulating cuff electrodes placed under the skin (left and right quadriceps, Q; left sartorius, Sart; right gastrocnemius and soleus, GS) or on pairs of silver hook electrodes placed in a paraffin oil pool (posterior biceps and semitendinosus, PBST; anterior biceps and semimembranosus, ABSM; gastrocnemius and soleus, GS, tibialis anterior and extensor digitorum longus branches jointly referred to as deep peroneal, DP; and cutaneous branches of the superficial peroneal, SP; all on the left side). A craniotomy over the cerebellum allowed for access to the lateral vestibular nucleus (LVN) and the brainstem reticular formation (RF) or the medial longitudinal fascicle (MLF). Laminectomies exposed the third to seventh lumbar segments (L3-L7) and lower thoracic segments (Th12Th13). A hemisection of the spinal cord was made at the thoracic level (Th12) contralateral to the reticular and vestibular stimulation sites to eliminate effects mediated by tract fibers that crossed either supraspinally or within the cervical or thoracic segments. The dura was left intact over the lumbar segments. Small holes were made in it to allow for the insertion of the recording microelectrodes. The exposed tissues were covered with warm liquid paraffin.

Stimulation and recording. MLF/RF and LVN were stimulated via tungsten electrodes insulated except for their tip (30-120 K $\Omega$ impedance), the indifferent electrode being inserted into neck muscles. Rectangular, constant current stimuli $0.2 \mathrm{msec}$ in duration were delivered, $50-100 \mu \mathrm{A}$ amplitude in MLF/RF and 50-200 $\mu \mathrm{A}$ amplitude in LVN. Single stimuli or trains of two to five stimuli (interval 3.3-6.6 msec) were used. Similar but thinner tungsten electrodes were used to stimulate axons of commissural interneurons in the contralateral motor nuclei. Peripheral nerves were stimulated with rectangular current pulses of 0.1 msec duration at strengths expressed in multiples of threshold $(T)$ for the most sensitive fibers, estimated on the basis of records of afferent volleys from the cord dorsum. Fibers in the left and right lateral funiculi at the level of the Th12-Th13 segments were stimulated transdurally via two pairs of silver ball-tipped electrodes, using stimuli of $0.2 \mathrm{msec}$ in duration, up to $500 \mu \mathrm{A}$.

Records from motoneurons were obtained by using glass micropipettes filled with 2 м potassium citrate solution (1.5-2 $\mu \mathrm{m}$ tip, 3-5 $\mathrm{M} \Omega$ resistance). Records from commissural neurons were made with either similar electrodes or electrodes filled with a mixture of $2 \%$ rhodamine dextran and Neurobiotin in $0.9 \%$ solution of $\mathrm{NaCl}$ (resistance, $15-20$ $\mathrm{M} \Omega$ ); the latter were used to label the neurons. Afferent volleys were recorded with a silver electrode in contact with the cord dorsum close to the entry zone of the left dorsal roots, $\sim 5-10 \mathrm{~mm}$ from the site of the micropipette insertion, the reference electrode being in contact with back muscles. Descending volleys were recorded at two sites. The first was at a Th12-Th13 level, with an electrode in contact with the ipsilateral (with respect to the RF and LVN stimulation sites) lateral funiculus. The second was at a lumbar level, with the same cord dorsum electrode that was used for recording afferent volleys, i.e., at the side of recording from motoneurons and commissural interneurons. The thoracic volleys were recorded primarily during the placement of the MLF and LVN electrodes and the lumbar volleys in parallel with the records from motoneurons and commissural interneurons.

Location of electrodes in the reticular formation and the lateral vestibular nucleus. The electrodes were placed in the brainstem ipsilaterally to interneurons and contralaterally to motoneurons recorded from. They were inserted at an angle of $30^{\circ}$ (tip directed rostrally) and positioned within MLF or in the adjacent part of RF (Horsley-Clarke coordinates: posterior 9-10, lateral $0.8-1.2$, horizontal -5.5 to -7.0 ) and in LVN (Horsley-Clarke coordinates: posterior $7.5-8.5$, lateral $4-5$, horizontal -3.5 to -4.5$)$. Descending volleys recorded from the thoracic cord (Th12-Th13) were used to adjust the final position of these electrodes. The electrodes were left at a depth from which descending volleys were evoked at a threshold of $10-20 \mu \mathrm{A}$ and at a latency of $\sim 2 \mathrm{msec}(2.2-2.8$ msec from LVN, 1.8-2.5 from MLF; the onsets of volleys evoked from the LVN were delayed by $0.3-0.4 \mathrm{msec}$ with respect to those evoked from the MLF at Th12-TH13 and by $0.4-0.5$ msec at L3-L5 levels; Fig. $1 D, E$ ). Identification of the LVN was assisted by initially recording antidromic field potentials (Ito et al., 1964; Grillner et al., 1970) (Fig. 1C) evoked by stimulation of vestibulospinal tract fibers in the thoracic segments, as described above, ipsilaterally to the brainstem electrodes. The placement of the electrodes subsequently was verified histologically by localizing small electrolytic lesions $(0.4 \mathrm{~mA}$ constant current for $15 \mathrm{sec})$ made at the end of the experiment. Fig. 1, $A$ and $B$, shows distribution of the stimulation sites, reconstructed from $100-\mu \mathrm{m}$-thick sections of the medulla, cut in the plane of the insertion of the electrodes.

Methodological problems. The reliability of our conclusions depends on two technical issues. The first of these concerns current spread from the MLF and LVN stimulation sites. Stimuli applied in MLF excite axons of a number of reticulospinal neurons, but they also might contribute to activation of some undefined reticulospinal and other brainstem neurons via axon collaterals of the stimulated fibers. The same stimuli also could excite neurons in the area lateral to MLF, considering that 50-100 $\mu \mathrm{A}$ stimuli would be effective up to a radius of $0.5-1.0 \mathrm{~mm}$ (Gustafsson and Jankowska, 1976). These complications could not be avoided, but control experiments have shown that both the characteristic RF descending volleys and the shortest latency EPSPs in contralateral motoneurons are evoked from only a very restricted part of MLF (Jankowska et al., 2003).

Stimuli applied in the region of the vestibular nuclei likewise could have evoked effects by exciting fibers passing through or close to the LVN in addition to axons of neurons around the tip of the stimulating electrode. Synaptic actions evoked via axon collaterals of these fibers as well as of fibers providing input to neurons in the vestibular nuclei could be another complicating factor. Particularly complicating would be actions of neurons in vestibular nuclei on reticulospinal neurons (see Peterson and Felpel, 1971; Maeda et al., 1975; Wilson and Peterson, 1978) and of neurons that activate both vestibulo- and reticulospinal neurons, e.g., those from the fastigial nucleus (Homma et al., 1995). For the interpretation of our results it is therefore important that, when the stimuli were applied at different depths in the area of the vestibular nuclei, only those 
A

P 8.0

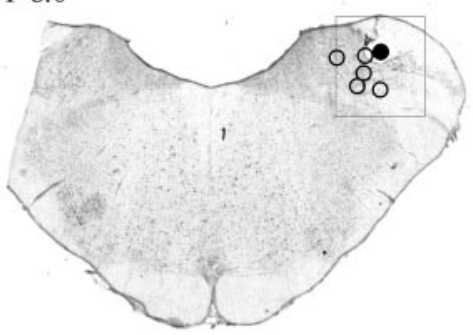

B

P 9.0

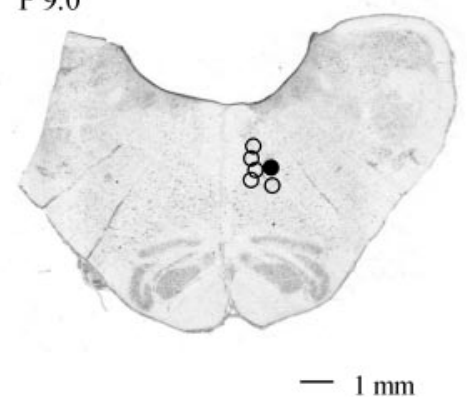

C

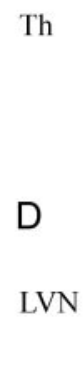

RF

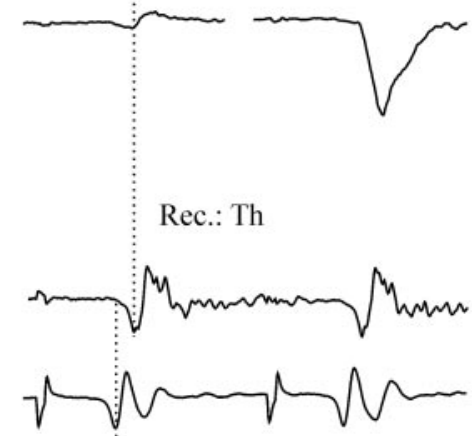

E

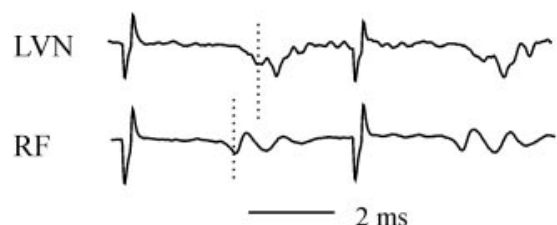

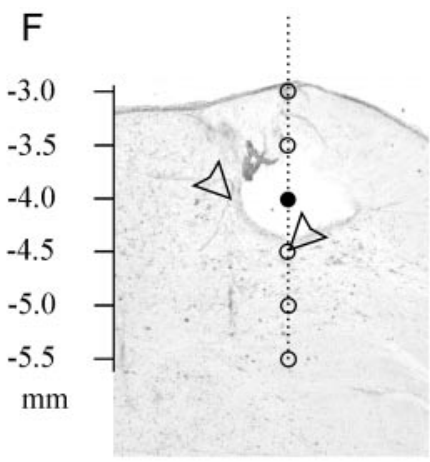

G

LVN $200 \mu \mathrm{A}$

$-3.0$

$-3.5$

$-4.0$

$-4.5$

$-5.0$

$-5.5$

$\mathrm{mm}$

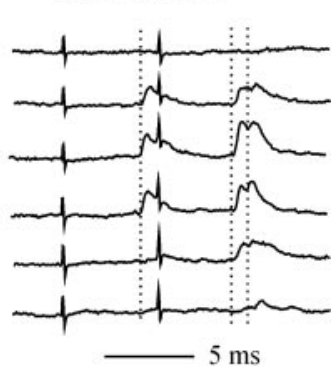

Figure 1. Brainstem stimulation sites. $A, B$, Stimulation sites in the regions of the LVN and MLF, superimposed on sections of the brainstem in which the electrodes were placed in the lateral part of the LVN and lateral to the MLF (open and filled circles). C, Records of field potentials (negativity upward) after stimulation of the ipsilateral lateral funiculus at the thoracic level, recorded at the two electrode positions indicated by the arrowheads in $F$. The LVN was stimulated at the more lateral site. D, Descending volleys recorded from the ipsilateral lateral funiculus at the Th12 level after paired stimulation of $L V N$ and $R F$ in the same experiment (at sites indicated by the filled circles in $A, B$ ). $E$, Descending volleys recorded from the ipsilateral lateral funiculus at the $L 5$ level after the same stimuli. Dotted vertical lines show the first positive peaks of the volleys. F, Boxed area in $A$ showing the electrolytic lesion in LVN. Arrowheads indicate sites of recording of field potentials shown in $C$; circles indicate sites of stimulation along an electrode track from which potentials shown in $G$ were evoked. G, EPSPs evoked in a commissural interneuron after LVN stimulation at different depths above, within, and below the LVN indicated in F. Dotted vertical lines indicate the monosynaptic and disynaptic components of the EPSPs. In this and the following figures the negativity is down in microelectrode records and up in records from the cord dorsum.

in a very restricted part of this area, which corresponded closely to that of the LVN nucleus (see Fig. $1 F, G$ ), were effective in inducing EPSPs in commissural interneurons or motoneurons. Stimulation of fibers in areas either dorsal or ventral to the nucleus was ineffective, although these regions might contain fibers that provide input to vestibulospinal neurons and axon collaterals of vestibular neurons. Effects of a mere spread of current between the MLF and LVN stimulation sites could be excluded by differences in effects evoked from these sites, the most marked being the much shorter latency of MLF effects. Furthermore, actions evoked by MLF stimuli were not reproduced even when stronger LVN stimuli (more likely to activate a larger number of reticulospinal tract fibers) were applied (see, for example, Fig. $3 B, C$ ). For these reasons effects of stimuli applied within MLF and LVN will be considered as being evoked primarily, although not necessarily exclusively, by reticulospinal and vestibulospinal tract fibers.

With respect to indirect (disynaptic and trisynaptic) actions, identification of the neurons that relay them is always a problem. In the preceding paper (Jankowska et al., 2003) we presented the results of control experiments that indicate that it is highly unlikely that supraspinal and long propriospinal neurons make a major contribution to disynaptic actions of reticulospinal tract fibers. The likelihood of contribution of such neurons to disynaptic and trisynaptic actions of vestibulospinal tract fibers will be taken up in Discussion. The second technical problem concerns the accuracy of latency measurements. The time resolution of our records was between 20 and $50 \mu \mathrm{sec}$, so the errors of the measurements of responses might amount to $\sim 0.1 \mathrm{msec}$. For this reason any latency differences to which we draw attention were at least $0.2 \mathrm{msec}$. The measurements of segmental latencies, i.e., latencies with respect to the time of arrival of descending volleys to the same segment, were also easier for PSPs of MLF than of LVN origin because the MLF volleys were much more synchronous and distinct, in particular at more caudal levels (Fig. $1 E)$. For this reason the latencies were measured from the shock artifacts and usually were related only subsequently to the first positive peak of descending volleys evoked from MLF or LVN at a lumbar level.

Sampling and data analysis. The sample of commissural neurons consisted of 20 intracellularly recorded cells located in the L4-L5 segments at depths (2.69-3.42 $\mathrm{mm}$ from the surface) at which distinct field potentials were evoked after MLF and LVN stimuli. For convenience of recording the interneurons were located on the left side of the spinal cord, the brainstem stimulation sites in these experiments being also on the left side. The neurons were identified as commissural interneurons by antidromic activation that followed stimuli $(10-50 \mu \mathrm{A})$ applied in the right GS motor nucleus in the L7 segment. The nucleus was located by recording antidromic field potentials that followed stimulation of the right GS nerve with a glass micropipette. Once the optimal angle and depth of the insertion had been found, the glass micropipette was replaced by the tungsten electrode and the records of the antidromic motor fields repeated. Neurons that were activated antidromically by stimulation of the lateral funiculi at the Th12-TH13 level were classified as ascending tract neurons and were excluded.

In total, 26 hindlimb motoneurons were sampled; all were identified by antidromic activation after stimulation of a muscle nerve. All of the motoneurons were located on the left side, with the brainstem stimulation at the right side and the thoracic hemisection at the left side. Both single responses and averages of 10-20 potentials were recorded and stored, using acquisition software designed by E. Eide, N. Pilgren, and T. Holmström (Department of Physiology, Göteborg University, Göteborg, Sweden). Measurements of latencies, amplitudes, and areas of the recorded potentials were made from averaged records. The spatial facilitation of reticulospinal and vestibulospinal actions was estimated, taking into account differences in the areas of PSPs evoked in motoneurons when the two descending systems were stimulated jointly and separately. Larger effects after combined stimulation of RF and LVN than the algebraic sum of those evoked after stimulation of either alone were taken as 


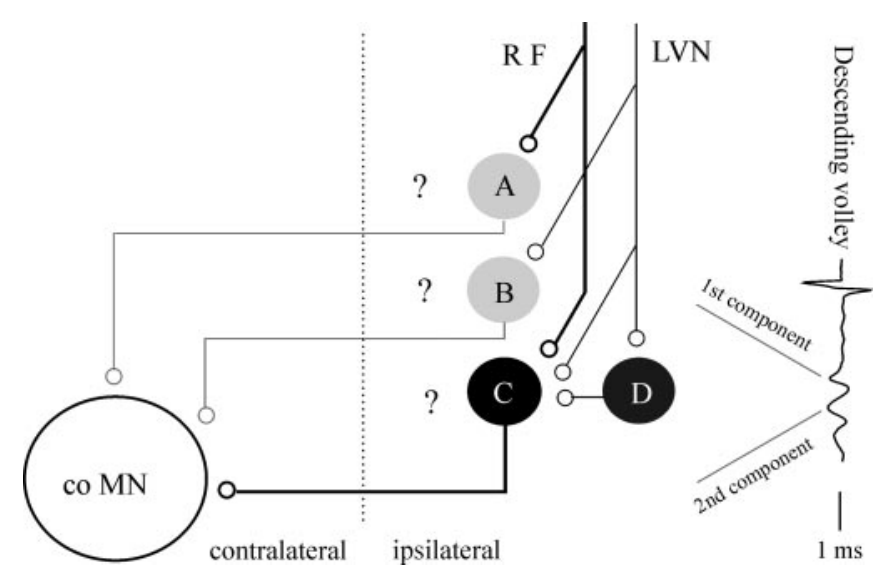

Figure 2. Diagram showing the possible arrangements for connections between the reticulospinal and vestibulospinal descending tract neurons and contralateral motoneurons via commissural interneurons. The gray circles indicate interneurons that mediate independent synaptic actions of either the reticulospinal $(A)$ or vestibulospinal $(B)$ neurons, whereas the black circle $(C)$ represents interneurons that are coexcited by the two descending systems, either directly or via additional interneurons $(D)$ as previously reported for neurons in upper cervical segments (Bolton et al., 1991). The dotted vertical line indicates the midline. Co MN, Contralateral motoneurons. The record of the descending volley after MLF stimulation (from Fig. 1E) indicates the origin of its first component (from axons of the descending fibers) and of its second component (from axons of interneurons that are excited by them).

the evidence of spatial facilitation. Statistical analysis was made with Student's $t$ test.

\section{Results}

Figure 2 shows diagrammatically how the reticulo- and vestibulospinal tract neurons might affect contralateral motoneurons: via separate premotor interneurons (Fig. $2 A, B$ ) or via shared interneurons (Fig. 2C). The existence of three populations of ventral horn interneurons with a selective or convergent input from RF and LVN has been demonstrated previously (Kozhanov and Shapovalov, 1977; Skinner and Remmel, 1978), but it has not been established to which of these populations commissural interneurons acting on contralateral motoneurons belong. Records from interneurons that project to contralateral motor nuclei could demonstrate directly whether or not such interneurons are coexcited by reticulo- and vestibulospinal neurons, and this was done first. However, this left open the question whether such interneurons indeed do act on motoneurons and whether they are excitatory or inhibitory. Therefore, recordings also were made from motoneurons to establish whether subthreshold MLF and LVN stimuli interact at a premotor level by spatial facilitation. If the two sources on input converge onto common interneurons, then combined subthreshold stimuli might discharge the interneurons producing EPSPs or IPSPs in motoneurons.

\section{Reticulospinal and vestibulospinal actions on commissural neurons}

Postsynaptic effects from MLF and LVN were compared in intracellular recordings from 20 midlumbar lamina VIII interneurons that were activated antidromically by stimuli delivered in the contralateral GS motor nucleus in the L7 segment. When the interneuron was recorded from extracellularly before penetration, antidromic activation was verified via the collision test by checking that the responses evoked from the motor nuclei were abolished by synaptically evoked spikes that preceded them within a critical interval, as illustrated in Figure $3 A$ (middle trace) and Figure $4 A$ (bottom trace). After penetration the spike gener- ation mechanism usually was damaged, but all-or-none blocked antidromic spikes that regularly appeared at a similar latency, as in Figure $3 B, D, E$ and in Figure $4, D$ and $E$, were recorded most often.

Commissural interneurons that could be activated from the GS motor nucleus belonged to several subpopulations, some with selective input from group II muscle or other afferents or from undefined sources. Of the three subpopulations of ventral horn interneurons reported previously (Kozhanov and Shapovalov, 1977; Skinner and Remmel, 1978), only two were represented: interneurons with selective input from RF and those with the convergent input from RF and LVN. For this reason the comparison of input from the reticulospinal and vestibulospinal tract fibers has been made on a population of commissural interneurons selected on the basis of monosynaptic input from RF.

The cells were concluded to be excited monosynaptically by reticulospinal neurons when EPSPs were evoked by MLF stimuli at a latency of $0.3-0.9 \mathrm{msec}$ from the first component of the descending volley recorded from the same segment and $<0.2$ msec from the second component. As previously demonstrated (Jankowska et al., 2003), these two components reflect spike activity in reticulospinal tract fibers and in spinal relay neurons activated by them, respectively, as indicated in Figure 2. Another requirement was that the EPSPs appeared after each successive stimulus of a train, with little temporal facilitation of the early components (Jankowska et al., 2003). Examples of such records are shown in Figure 3, $B$ and $D$, and Figure 4, $B$ and $D$. Latencies of $\geq 1 \mathrm{msec}$ and clear-cut temporal facilitation defined the EPSPs as evoked disynaptically.

Stimulation of LVN evoked EPSPs in $80 \%$ (16 of 20) of the interneurons that were analyzed. In seven of them (35\% of the total sample) the properties of the EPSPs were characteristic of a disynaptic linkage; the EPSPs were evoked at segmental latencies of 1.1-1.8 msec and displayed temporal facilitation. An example is shown in Figure $3 C$, in which EPSPs followed by IPSPs appeared only after the third LVN stimulus (top trace), and even a much stronger initial stimulus evoked only a very small EPSP (with the onset indicated by the second dotted line) that grew substantially in amplitude after the second and third stimuli (middle trace). The latencies of these EPSPs with respect to stimulus artifacts exceeded latencies of EPSPs evoked from MLF that were classified as monosynaptic by $1.11 \pm 0.16 \mathrm{msec}$ (mean and SEM, statistically significant at $<0.01$; range, $0.6-1.6 \mathrm{msec}$ ), whereas LVN volleys were delayed with respect to MLF volleys by only $\sim 0.5 \mathrm{msec}$. There was no statistically significant difference between latencies of temporally facilitated EPSPs or IPSPs (with an example in Fig. $3 E$ ) of LVN origin and latencies of disynaptic components of EPSPs or IPSPs of RF origin. The latter are illustrated in Figure $3 D$ (third dotted line after the second stimulus) and in Figure $4 D$ (third dotted line after the third stimulus).

In nine other interneurons ( $45 \%$ of the sample) LVN stimuli were much more effective in evoking EPSPs because, as illustrated in Figure 4, $C$ and $E$, these EPSPs were induced by the first stimulus and their early components showed little or no temporal facilitation, suggesting that they were evoked monosynaptically. However, the latencies of only four of these were in keeping with this possibility. They were $0.7-0.9 \mathrm{msec}$ from earliest components of the descending volleys and only $0.2-0.5 \mathrm{msec}$ longer than segmental latencies of monosynaptic EPSPs of MLF origin in the same neurons. In the remaining five cells latencies of the EPSPs overlapped with latencies of EPSPs that displayed temporal facilitation (1.1-1.3 msec from the descending volleys; $0.9-1.1$ msec longer than latencies of monosynaptic EPSPs of MLF origin 

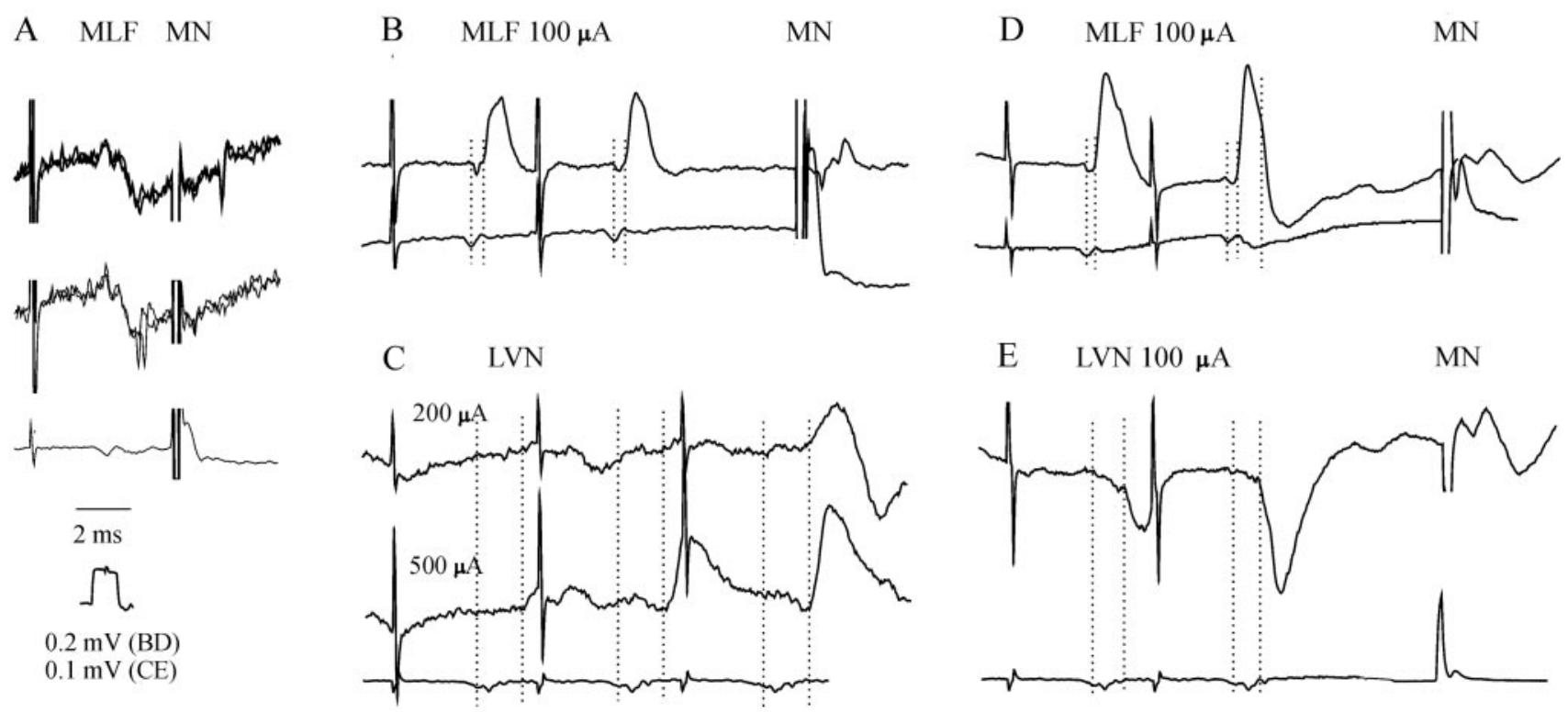

Figure 3. Examples of disynaptic synaptic actions from the LVN in two commissural interneurons with monosynaptic EPSPs from MLF. $A-E$, Records from two interneurons. $A$, Extracellular records (top and middle; two superimposed traces) and cord dorsum potentials (bottom trace) of collision between spike potentials from motor nucleus (MN) and spikes from the reticular formation (MLF). $B-E$, Top traces and middle trace in ( are averaged intracellular records $(n=10)$ of EPSPs and/or IPSPs after successive MLF or LVN stimuli and of blocked antidromic spikes from the motor nucleus, as indicated. Bottom traces are simultaneously recorded cord dorsum potentials. Dotted vertical lines indicate the positive peaks of the early components of the descending volleys seen in cord dorsum records and the onsets of the PSPs. Time and voltage calibrations in $A$ apply to all records. The largest shock artifacts are truncated.
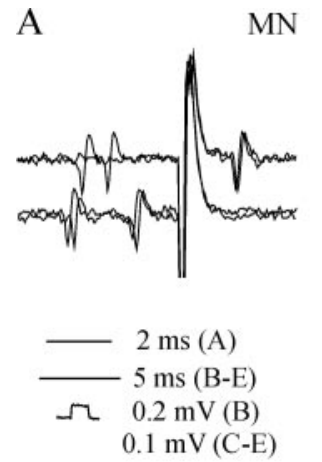

B $\quad$ MLF $100 \mu \mathrm{A}$

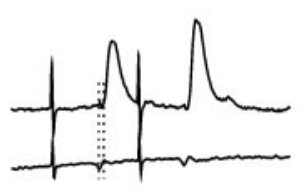

C $\quad \operatorname{LVN} 200 \mu \mathrm{A}$

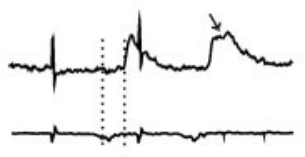

D MLF $100 \mu \mathrm{A}$

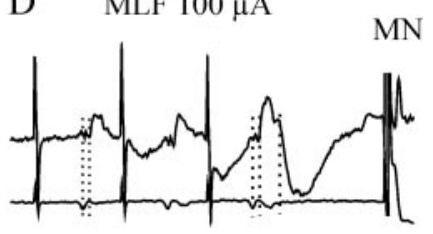

E $\quad$ LVN $100 \mu \mathrm{A} \quad$ MN

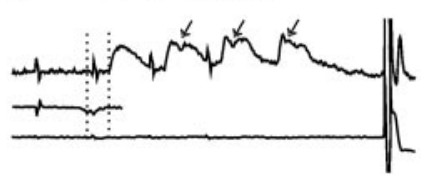

Figure 4. Examples of synaptic actions from LVN that most likely were evoked both monosynaptically and disynaptically. $A-E$, Records from two commissural interneurons. In $A$ all records are extracellular and illustrate the collision between the synaptically and antidromically evoked responses. In $B-E$ the top traces are intracellular records and the bottom traces are from the cord dorsum. The additional cord dorsum record in $E$ shows descending volleys induced by the first LVN stimulus at a more rostral level at a higher gain. Other conventions are as in Figure 3 .

from stimulus artifacts). They thus might be compatible with either monosynaptic or disynaptic coupling (see Discussion). As judged by temporal facilitation of the later components of EPSPs of LVN origin (arrows in Fig. 4C,D) evoked in apparently monosynaptically excited interneurons, additional interneurons also would provide disynaptic input to them. Similar differences in amplitudes of EPSPs evoked from MLF (larger) and from LVN (smaller), as in cells illustrated in Figures 3 and 4, were seen in all of the interneurons.

\section{Mutual facilitation of reticulospinal and vestibulospinal actions on contralateral motoneurons}

According to the original experimental design, the intention was to select motoneurons on the basis of the presence of oligosyn- aptic input from either MLF or LVN. However, special measures usually were needed to disclose input from LVN (longer trains of stimuli, higher stimulus intensities, spatial facilitation of effects of LVN and MLF stimuli; see below). The motoneurons therefore were selected on the basis of their input from MLF (EPSPs or IPSPs of at least $0.5 \mathrm{mV}$ ). The sample of 26 motoneurons that were analyzed included 13 Q, 2 GS, 2 PBST, and 1 unidentified motoneuron with short-latency EPSPs from MLF and 8 Sart motoneurons with short-latency IPSPs from MLF.

\section{Facilitation of disynaptic EPSPs}

The earliest components of EPSPs evoked from MLF apparently were induced disynaptically because they appeared at segmental latencies of $1.33 \pm 0.04$ msec (mean \pm SE; range, $1.0-1.7 \mathrm{msec} ; n=18$; Fig. $5 A$ ), which would be too long for monosynaptic actions and too short for trisynaptic actions (for discussion, see Jankowska et al., 2003).

EPSPs from LVN were found in 35\% (9 of 26) of the total sample of the motoneurons. However, they were found in a somewhat larger $(50 \%)$ proportion of the 18 motoneurons in which EPSPs were evoked from MLF. Four or five stimuli of 200 $\mu \mathrm{A}$ in a train usually were needed to evoke EPSPs from LVN. Only in two motoneurons were they evoked after three stimuli at $100 \mu \mathrm{A}$ (Figs. 5F, 7B). In none of the penetrated motoneurons was selective input from LVN detected with trains of four $200 \mu \mathrm{A}$ of stimuli.

Temporal characteristics of EPSPs evoked from LVN were similar to characteristics of EPSPs evoked from MLF. The LVN EPSPs were evoked at segmental latencies of $1.73 \pm 0.05 \mathrm{msec}$ 
(range, $1.4-2.1 \mathrm{msec} ; n=9$; Fig. $5 B$ ), overlapping with, but most longer than, the latencies of EPSPs of MLF origin. Differences between segmental latencies of LVN and MLF EPSPs (Fig. 5C) did not exceed $0.35 \mathrm{msec}$ in six motoneurons, one of which is illustrated in Figure 5F, indicating that the LVN EPSPs also were evoked disynaptically. However, in three other motoneurons the segmental latencies of LVN EPSPs exceeded those of RF EPSPs by $0.4-$ $0.5 \mathrm{msec}$, which might be more compatible with a trisynaptic linkage. These observations are thus in keeping with the previous evidence that LVN neurons evoke disynaptic EPSPs in some contralateral motoneurons (Hongo et al., 1971, 1975). Temporal facilitation at a premotoneuronal level often was needed to induce EPSPs in motoneurons from either MLF (Figs. $6 A, 7 D$ ) or LVN (Fig. $5 F$ ), as found in previous studies (Shapovalov, 1969; Hongo et al., 1971, 1975; Maeda et al., 1975; Jankowska et al., 2003). Using this property, we investigated convergence from MLF and LVN at the level of commissural neurons by combining temporal facilitation with spatial facilitation. To this end a single subthreshold RF stimulus was added to the end of a train of similarly subthreshold or nearthreshold LVN stimuli, or vice versa. Thereby it was expected to be easier to demonstrate that the joint effect of the two stimuli exceeded the sum of effects evoked by them independently (Lundberg, 1979). Facilitation obtained in this way is illustrated in Figure $6 A-C$. Records in Figure 6, $A$ and $B$, show that disynaptic EPSPs were evoked by two relatively strong $(100 \mu \mathrm{A}) \mathrm{RF}$ stimuli, whereas no EPSPs were evoked when single weaker MLF stimuli or double LVN stimuli were applied. However, when these subthreshold MLF and LVN stimuli were applied jointly, with the MLF stimuli preceding or following the last LVN stimuli, the EPSPs appeared (Fig. 6C). At an optimal interval the facilitated EPSP in Figure $6 \mathrm{C}$ reached nearly one-half of the EPSP evoked by the second MLF stimulus in Figure $6 \mathrm{~A}$.

MLF stimuli were usually the most effective, so single MLF stimuli were used most frequently as test stimuli after a train of conditioning LVN stimuli, as illustrated in Figure $6 C$. The reverse order was used in two of 18 motoneurons, those in which substantial disynaptic EPSPs were evoked from LVN. Records from one of these are illustrated in Figure 7. EPSPs evoked by three suprathreshold MLF and LVN stimuli are shown in Figure 7, A and $B$, and the failure of single LVN stimuli to evoke any EPSPs in Figure 7C. However, when single LVN stimuli were applied instead of the third MLF stimuli, they evoked EPSPs (compare Fig. $7 D, E$ within the boxed areas and the superimposed expanded parts of these records in F). EPSPs that followed single LVN stimuli had the time characteristics of EPSPs evoked by the third LVN stimuli and appeared at the same latency (compare EPSPs that follow the second dotted line in Fig. $7 B, E$ ), indicating facilitation of activation of interneurons mediating LVN actions by MLF stimuli.

In total, joint application of near-threshold or subthreshold MLF and LVN stimuli resulted in the appearance of EPSPs or in a considerable increase in amplitude of EPSPs evoked by separate application of somewhat stronger stimuli in all of the $18 \mathrm{mo}-$ toneurons that were tested. These observations thus lead to the conclusion that commissural interneurons with input from both MLF and LVN mediate excitation of a considerable proportion of, if not all, contralateral motoneurons.

\section{Facilitation of disynaptic IPSPs}

In four of eight Sart motoneurons the IPSPs were evoked not only by MLF stimuli but also by LVN stimuli when these were applied alone. Segmental latencies of IPSPs from MLF were 1.3-1.7 msec (Fig. 5D) and were fully compatible with disynaptic coupling (Jankowska et al., 2003). Segmental latencies of IPSPs from LVN were 1.9-2.1 msec (Fig. 5E), indicating that they were evoked either disynaptically or trisynaptically, as were the EPSPs. The IPSPs required temporal facilitation of the synaptic actions of two or more stimuli at a premotoneuronal level and were evoked more readily from MLF than from LVN.

Joint application of subthreshold MLF and LVN stimuli evoked IPSPs in all eight motoneurons that were tested. This is illustrated in Figure 8 with records from a motoneuron in which a disynaptic IPSP was evoked only after the third MLF stimulus (Fig. $8 A$ ), whereas single MLF stimuli (Fig. $8 B$ ) and four LVN stimuli (Fig. $8 C$ ) were ineffective when they were applied separately. However, single MLF stimuli applied just before or after the fourth LVN stimulus resulted in the appearance of distinct disynaptic IPSPs (Fig. 8D).

\section{Optimal intervals between conditioning and test stimuli}

The records in Figures $6 C$ and $8 D$ show that the degree of facilitation of both EPSPs and IPSPs depended on the time interval between the descending volleys that followed the test and conditioning stimuli. In an attempt to quantify this relationship, we have plotted the amplitudes (areas) of the conditioned EPSPs against time intervals between the descending volleys after the test stimulus and the last conditioning stimulus (Fig. 6D,E). The 
A

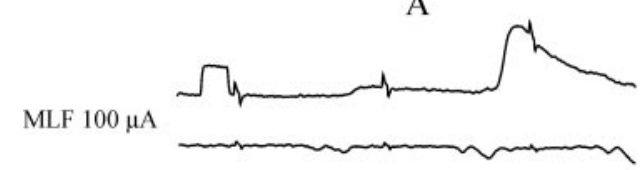

B

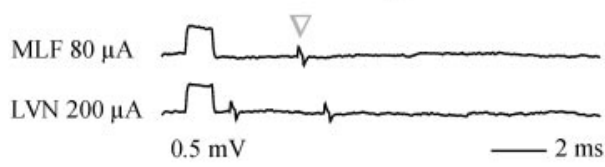

$\mathrm{C}$

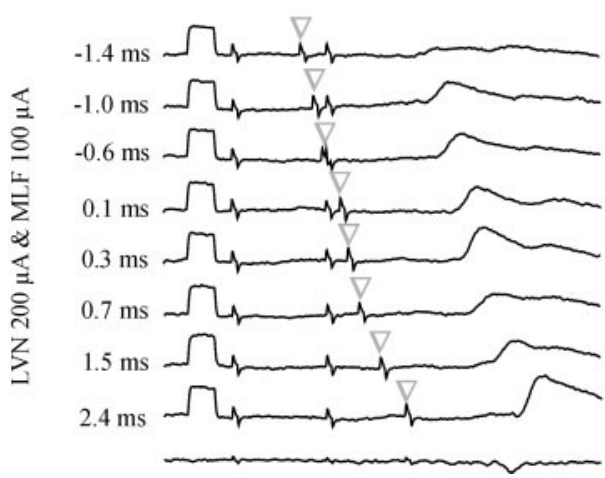

D
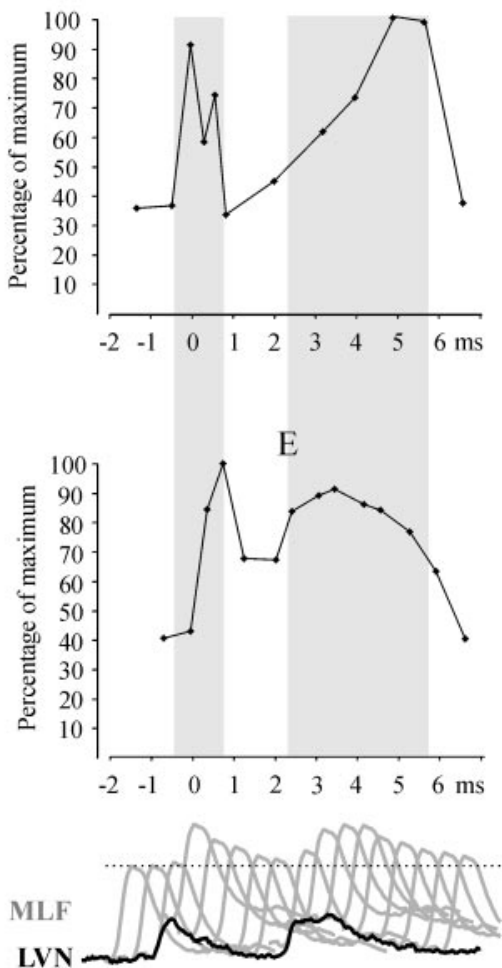

Figure 6. Facilitation of EPSPs of MLF origin by LVN stimuli. $A-C$, Records from a GS motoneuron with corresponding cord dorsum potentials (bottom traces in $A, C$ ). $A$, Effects of two supra-threshold MLF stimuli. $B, A$ demonstration that a weaker single MLF stimulus (indicated by an arrowhead) and double LVN stimuli were both subthreshold. C, Facilitation of effects of these subthreshold stimuli when MLF stimuli (arrowheads) were applied before or after the second LVN stimuli. The figures to the left indicate intervals between the early components of MLF and LVN descending volleys. D, E, Changes in the size (ordinate) of the EPSPs evoked in a PBST and a Q motoneuron after MLF stimuli as a function of intervals between the MLF and LVN volleys (abscissa). The volleys coincided at a time 0 ; negative and positive values are for intervals at which the MLF volleys preceded and followed the last LVN volleys, respectively. The sizes of the EPSPs evoked in the two motoneurones at different intervals are expressed as percentages of the areas within 1.2 and 2 msec from the onset of the maximal facilitated MLF EPSPs (taken as 100\%), respectively. Records below $E$ consider the likely summation of single EPSPs evoked from MLF (gray) and of two EPSPs evoked by double LVN stimuli 3.3 msec apart (black) in a commissural interneuron (using EPSPs from Fig. $4 B, C$ ) at different time intervals between the MLF and LVN stimuli. The dotted horizontal line indicates a hypothetical threshold for the generation of action potentials in this commissural interneuron. The gray columns indicate periods of time during which the spatial facilitation was most effective.

areas were measured from 1-3 msec time windows after the onset of the most distinct facilitated EPSPs. Longer time windows were chosen for EPSPs with the longest time-to-peak and lowest amplitudes to increase the reliability of the measurements, but confining them to the early components of the EPSPs. The areas of EPSPs evoked at different conditioning-testing intervals then have been related to the maximal facilitated EPSPs (taken as $100 \%)$. Plots for two of the 10 motoneurons tested in this way in one experiment are shown in Figure $6, D$ and $E$. These plots illustrate three main features of the relationship between the degree of facilitation of the EPSPs and the timing of the test and conditioning stimuli. First, the strongest facilitation occurred within the two time periods indicated by the gray columns: when the test descending volleys preceded or followed the last conditioning descending volleys by $\sim 1 \mathrm{msec}$ and when the test descending volleys were delayed with respect to the last conditioning volleys by $\sim 2-4 \mathrm{msec}$. Second, the duration of the facilitation was shorter during the first period, whether the size of the EPSPs returned to the original one (as in Fig. 6D) or continued to exceed it (Fig. $6 E$ ) between the two periods. Third, the total duration of the facilitation associated with double-conditioning stimuli was $\sim 5$ msec. Similar data were obtained for EPSPs in all 10 motoneurons that were tested as well as for IPSPs. The latter are illustrated in Figure 8.

An explanation of the two periods of facilitation and its long duration is proposed by considering the effects of LVN and RF stimuli at the level of the individual commissural interneurons responsible for the PSPs evoked in motoneurons. The black EPSPs in Figure $6 E$ represent EPSPs evoked by two LVN stimuli in such an interneuron. As described above (Fig. 4B,C), the first most likely was evoked predominantly monosynaptically and the second both monosynaptically and disynaptically and therefore displayed a slower declining phase. The gray EPSPs represent EPSPs evoked by MLF stimuli that preceded, coincided with, or followed LVN stimuli at intervals of $0.5 \mathrm{msec}$. Depending on these intervals, either the sums of EPSPs evoked by the test and conditioning stimuli would remain subthreshold for generating an action potential (represented by the horizontal dotted line) or they would discharge the interneuron, which, in turn, would induce an EPSP in motoneurons. As suggested by this hypothetical reconstruction, the action potential threshold would be reached when the peak of the MLF-evoked EPSPs coincides with the rising or declining phase of EPSPs evoked from LVN, especially with their peak. The probability of induction of action potentials of the interneuron, accordingly, would be highest at conditioning testing intervals that coincide with those indicated by the gray columns in Figure 6, D and E. Depending on the time characteristics of EPSPs evoked by successive conditioning stimuli in the population of commissural interneurons synapsing with a given motoneuron and the size of the population, the probability of discharging these interneurons and of their actions on motoneurons would increase for shorter or longer period of times. An increase in the duration of EPSPs when disynaptic components are added to their monosynaptic components after temporal facilitation by successive stimuli may explain further the need of trains of such stimuli and the importance of disynaptic excitation of the interneurons for their activation.

\section{Discussion}

The results of this study show that a considerable proportion of midlumbar commissural interneurons that project to contralateral motor nuclei is coexcited by reticulospinal and vestibulospinal tract fibers. These therefore may be used to adjust the activation of contralateral limb muscles by joint actions from both the vestibulospinal and reticulospinal systems. Mutual facilitation of both EPSPs and IPSPs evoked from LVN and MLF in contralateral motoneurons shows, in addition, that the joint actions of vestibulospinal and reticulospinal neuronal systems are exerted not only by excitatory but also by inhibitory commissural neurons and reach a very high proportion of motoneurons. 


\section{Coupling between vestibulospinal neurons and commissural neurons with monosynaptic RF input}

In terms of the hypothetical neuron circuitry indicated in Figure 2, we have positive evidence that commissural interneurons coexcited by reticulospinal and vestibulospinal neurons (of the type labeled C) do exist. We also have strong indications for neurons of type $\mathrm{D}$, interposed between vestibulospinal neurons and interneurons $\mathrm{C}$, and for a smaller proportion of interneurons of type A, with selective input from reticulospinal tract neurons, but not from vestibulospinal tract neurons. We have not found interneurons of type B, with monosynaptic EPSPs from LVN, but not from MLF (Kozhanov and Shapovalov, 1977; Skinner and Remmel, 1978). However, we cannot conclude that interneurons with a selective LVN input have exclusively ipsilateral actions on this basis. Commissural interneurons with a selective LVN input could, for instance, project to other contralateral motor nuclei or be located in segments that were not explored in this study. They might, for example, be located preferentially in the rostral lumbar segments where the terminal branching of vestibulospinal fibers is particularly dense (Kuze et al., 1999). Excitation of such neurons also could be too weak under our experimental conditions to allow us to recognize them in extracellular recording before any attempts to penetrate them.

In only four of the 20 commissural interneurons of the present sample did the EPSPs of LVN origin fulfill both of the main criteria of monosynaptically evoked EPSPs: following consecutive stimuli in a train without or with little temporal facilitation and appearing at latencies of $<1 \mathrm{msec}$ from the onset of the descending volleys. The latencies of these EPSPs were in the same range as EPSPs in a number of previously investigated ventral horn interneurons excited by LVN neurons (Aoyama et al., 1971; Skinner and Remmel, 1978; Davies and Edgley, 1994). Five other interneurons fulfilled the first criterion, although their segmental latencies exceeded $1.1 \mathrm{msec}$ and were $\sim 0.6 \mathrm{msec}$ longer than segmental latencies of EPSPs evoked from RF. They were thus difficult to classify with confidence because they might have been evoked either by very secure disynaptic connections or by monosynaptic connections from slower-conducting vestibulospinal neurons. Monosynaptic actions at longer latencies also could reflect trans-synaptic rather than direct activation of some fastconducting vestibulospinal neurons in the brainstem, if stimuli applied in the LVN activated presynaptic fibers, which, in turn, excited LVN neurons and LVN neurons monosynaptically excited commissural neurons. Depending on the reasons for the longer latencies of these EPSPs, the proportion of commissural neurons with monosynaptic input from RF in which monosynaptic EPSPs are evoked from LVN thus might have been between 20 and $45 \%$ of neurons of the present sample.

In contrast, EPSPs evoked from the LVN in the remaining 11 commissural interneurons had typical features of disynaptically evoked PSPs because they were evoked at longer latencies and also required temporal facilitation. Later temporally facilitated components also were seen after the apparently monosynaptically evoked EPSPs (Fig. 4C,E). The majority of the commissural
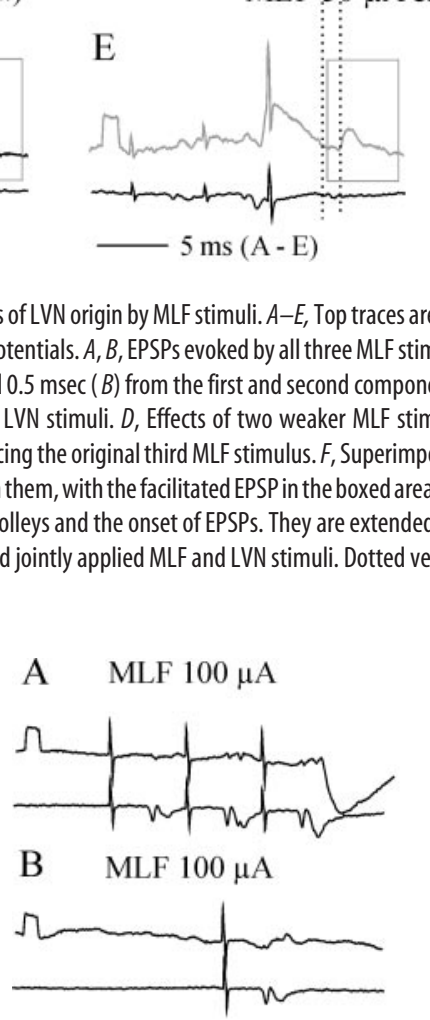

$5 \mathrm{~ms}(\mathrm{~A}-\mathrm{E})$

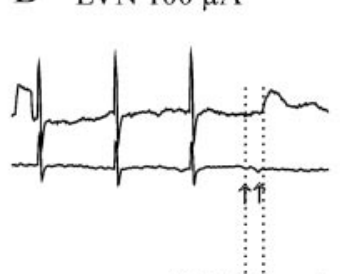

C $\operatorname{LVN} 100 \mu \mathrm{A}$ (test)
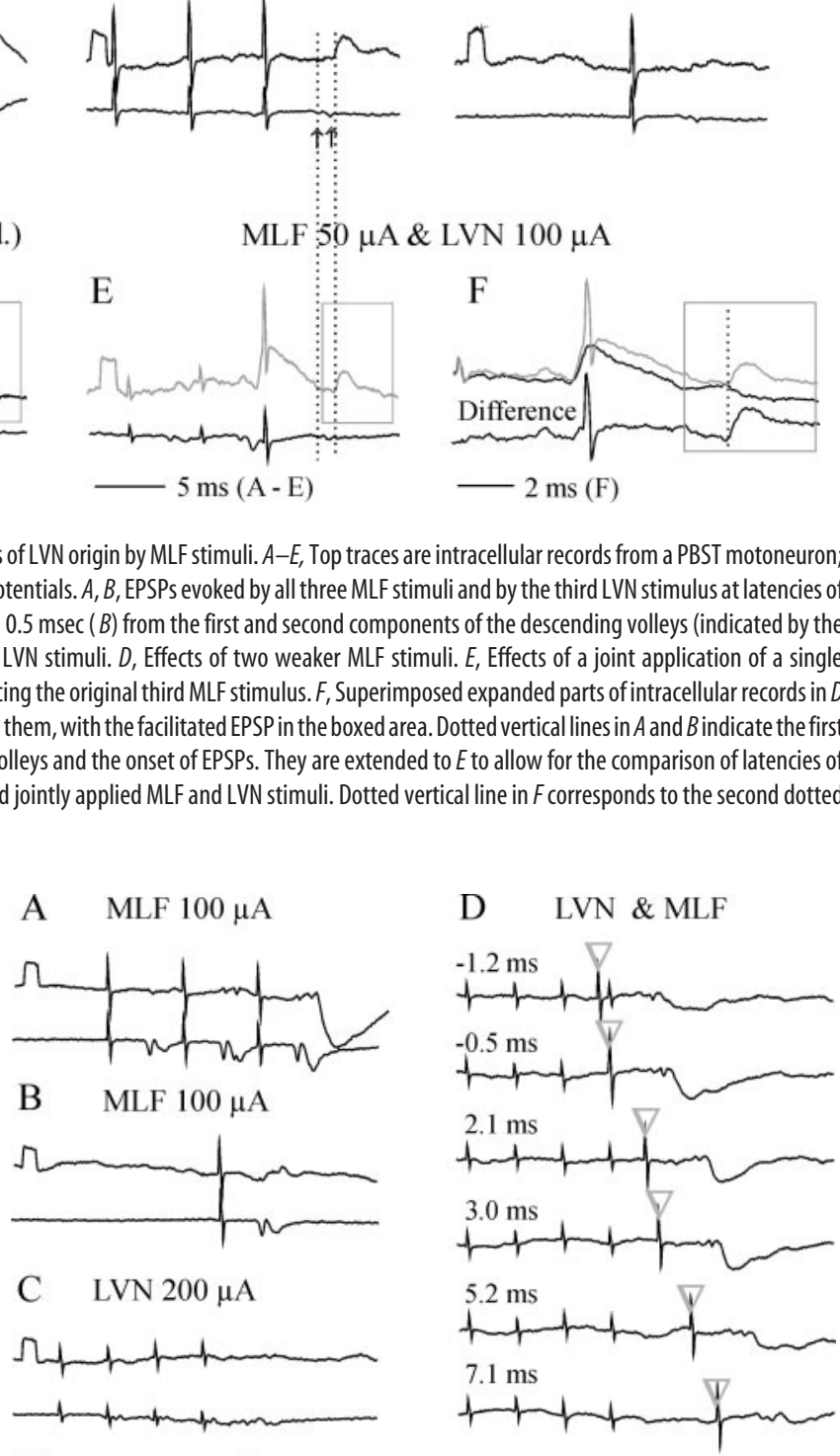

$0.2 \mathrm{mv}$ $-5 \mathrm{~ms}$

Figure 8. Facilitation of IPSPs of RF origin by LVN stimuli. A-C, Top records are from a Sart motoneuron; bottom records are from the cord dorsum. $A$, IPSPs after the third MLF stimulus of a train. $B, C$, Failures of single MLF stimuli and a train of four LVN stimuli to evoke an IPSP. D, Intracellular records from the same motoneuron showing IPSPs evoked by joint actions of the stimuli from $B$ and $C$ when both were combined. The timing of the MLF stimuli is indicated by arrowheads; they preceded, coincided with, or followed the last LVN stimulus of the train. Time differences between the first components of the descending volleys evoked by these stimuli are shown above the records.

interneurons that were investigated thus appeared to be excited disynaptically by LVN neurons.

The proportion of commissural neurons that were excited either monosynaptically or disynaptically by MLF and LVN neurons appeared to be larger $(80 \%)$ than of ipsilaterally projecting intermediate zone interneurons $(\sim 50 \%)$ (Davies and Edgley, 1994), but proportions of monosynaptically coexcited commissural interneurons $(20-45 \%$ in the present study) and of the previously investigated undefined ventral horn interneurons ( 25\%) (Skinner and Remmel, 1978) might be comparable. Taking monosynaptic as well as disynaptic LVN input to commissural neurons into account, both disynaptic and trisynaptic 
EPSPs or IPSPs would be expected in motoneurons contacted by commissural interneurons but with the trisynaptic PSPs dominating. In agreement with these expectations, EPSPs fulfilling criteria of disynaptically evoked EPSPs have been found in contralateral motoneurons, both previously (Hongo et al., 1971, 1975) and in the present study, but most of the EPSPs and some of the IPSPs were evoked at longer latencies and apparently trisynaptically rather than disynaptically, i.e., by two rather than one interneuron in series.

Which neurons mediate disynaptic excitation of commissural interneurons from LVN is an open question. The disynaptic LVN actions could be relayed supraspinally, for example, via reticulospinal neurons or via neurons located within the cervical or thoracic segments, if these were excited by axon collaterals of neurons stimulated in the vestibular nuclei or in their vicinity. We know of no experimental indications to support or to refute the involvement of trans-synaptically excited reticulospinal neurons, but such an involvement would be possible in view of a coupling between vestibulospinal and reticulospinal neurons (Peterson and Felpel, 1971; Maeda et al., 1975; Wilson and Peterson, 1978). Vestibulospinal tract neurons also have been shown to provide input to another population of neurons that might excite commissural interneurons. These are cervical long propriospinal neurons with input from both RF and LVN (Alstermark et al., 1987b), some of which are involved in mediating tonic neck reflexes (Brink et al., 1985).

However, we postulate that at least some neurons mediating disynaptic inhibition of commissural interneurons are among spinal interneurons interposed between vestibulospinal fibers and commissural interneurons (as interneuron D in Fig. 2) for two reasons: first, because disynaptic excitation of another subpopulation of commissural interneurons has been demonstrated to be mediated by spinal interneurons (S. A. Edgley, E. Jankowska, P. Krutki, and I. Hammar, unpublished data) and, second, because amplitudes of both disynaptic and later components of EPSPs of LVN origin evoked in ipsilateral motoneurons were shown to be modulated during the locomotor cycle, with indications that they were mediated by spinal interneurons (Gossard et al., 1996). Because modulation of the activity of ipsilateral and contralateral motoneurons during locomotion is tightly related, it may be justified to extend this conclusion to interneurons mediating synaptic actions of vestibulospinal tract neurons to contralateral motoneurons. Whichever way disynaptic excitation of commissural interneurons by vestibulospinal tract fibers is mediated, these interneurons may be considered as mediating both disynaptic and trisynaptic LVN actions on contralateral motoneurons. Trisynaptic actions of LVN origin nevertheless also could be mediated by contralaterally located interneurons interposed between the commissural interneurons and the motoneurons, because the terminal branching areas of some commissural neurons have been found outside the contralateral motor nuclei (Bannatyne et al., 2003; K. Matsuyama, personal communication) within regions in which ipsilaterally projecting premotor interneurons can be located.

\section{Functional consequences of both monosynaptic and disynaptic LVN input to commissural neurons}

Parallel monosynaptic and disynaptic LVN input to commissural interneurons is reminiscent of the recently described parallel monosynaptic and disynaptic input from group II muscle afferents to intermediate zone premotor interneurons (Jankowska et al., 2003). The consequences of this arrangement might be considered in similar terms, i.e., to allow for independent modula- tion of LVN synaptic actions at the level of the last-order commissural interneurons and at the level of other interneurons with LVN input. However, in contrast to the network of interneurons in pathways from group II afferents, too little is known about subpopulations of interneurons with LVN input (e.g., about specific segmental or supraspinal input and neuronal systems that might modulate their activity) to predict their relative role in the network of neurons mediating actions of vestibulospinal tract neurons on contralateral motoneurons. It is, for example, not known whether input from different semicircular canals, otoliths, and neck receptors (Brink et al., 1985; Kasper et al., 1988, 1989; Wilson et al., 1990; Wilson and Schor, 1999) is distributed similarly to various spinal interneurons and whether their activation may be modulated independently.

The LVN input to commissural neurons appeared to be weaker than the MLF input as judged by amplitudes of EPSPs recorded in the same interneurons (Figs. 3-5) and by the lower probability of finding commissural interneurons activated by LVN stimuli in extracellular records (however, see above). LVN actions on commissural interneurons thus might depend on activation of the additional interneurons ( $\mathrm{D}$ in Fig. 2) by other neuronal systems to a much greater extent than MLF actions. As stressed by Wilson and Peterson (1978), the presence of spinal interneurons in pathways from LVN means that the effectiveness of these pathways can be enhanced or diminished by changing their excitability, and interactions between the vestibulospinal and other neuronal systems on D neurons may serve this purpose. However, as pointed out above, the disynaptic input to commissural interneurons could be provided by several neuronal populations, all of which may be involved in securing their activation.

\section{References}

Alstermark B, Lundberg A, Pinter M, Sasaki S (1987a) Long C3-C5 propriospinal neurones in the cat. Brain Res 404:382-388.

Alstermark B, Lundberg A, Pinter M, Sasaki S (1987b) Vestibular effects in long C3-C5 propriospinal neurones. Brain Res 404:389-394.

Alstermark B, Lundberg A, Pinter M, Sasaki S (1987c) Subpopulations and functions of long C3-C5 propriospinal neurones. Brain Res 404:395-400.

Aoyama M, Hongo T, Kudo N, Tanaka R (1971) Convergent effects from bilateral vestibulospinal tracts on spinal interneurons. Brain Res 35:250-253.

Bannatyne BA, Maxwell DJ, Edgley SE, Hammar I, Jankowska E (2003) Commissural interneurons in cat spinal motor pathways: identification of excitatory and inhibitory cells. J Physiol (Lond) 548P:P116.

Bolton PS, Goto T, Wilson VJ (1991) Commissural neurons in the cat upper cervical spinal cord. NeuroReport 2:743-746.

Brink EE, Suzuki I, Timerick SJ, Wilson VJ (1985) Tonic neck reflex of the decerebrate cat: a role for propriospinal neurons. J Neurophysiol 54:978-987.

Davies HE, Edgley SA (1994) Inputs to group II-activated midlumbar interneurones from descending motor pathways in the cat. J Physiol (Lond) 479:463-473.

Floeter MK, Sholomenko GN, Gossard JP, Burke RE (1993) Disynaptic excitation from the medial longitudinal fasciculus to lumbosacral motoneurons: modulation by repetitive activation, descending pathways, and locomotion. Exp Brain Res 92:407-419.

Gossard JP, Floeter MK, Degtyarenko AM, Simon ES, Burke RE (1996) Disynaptic vestibulospinal and reticulospinal excitation in cat lumbosacral motoneurons: modulation during fictive locomotion. Exp Brain Res 109:277-288.

Grillner S, Hongo T (1972) Vestibulospinal effects on motoneurones and interneurones in the lumbosacral cord. Prog Brain Res 37:243-262.

Grillner S, Lund S (1968) The origin of a descending pathway with monosynaptic action on flexor motoneurones. Acta Physiol Scand 74:274-284.

Grillner S, Hongo T, Lund S (1968) Reciprocal effects between two descending bulbospinal systems with monosynaptic connections to spinal motoneurones. Brain Res 10:477-480. 
Grillner S, Hongo T, Lund S (1970) The vestibulospinal tract. Effects on $\alpha$-motoneurones in the lumbosacral spinal cord in the cat. Exp Brain Res 10:94-120.

Grillner S, Hongo T, Lund S (1971) Convergent effects on $\alpha$-motoneurones from the vestibulospinal tract and a pathway descending in the medial longitudinal fasciculus. Exp Brain Res 12:457-479.

Gustafsson B, Jankowska E (1976) Direct and indirect activation of nerve cells by electrical pulses applied extracellularly. J Physiol (Lond) 258:33-61.

Homma Y, Nonaka S, Matsuyama K, Mori S (1995) Fastigiofugal projection to the brainstem nuclei in the cat: an anterograde PHA-L tracing study. Neurosci Res 23:89-102.

Hongo T, Kudo N, Tanaka R (1971) Effects from the vestibulospinal tract on the contralateral hindlimb motoneurones in the cat. Brain Res 31:220-223.

Hongo T, Kudo N, Tanaka R (1975) The vestibulospinal tract: crossed and uncrossed effects on hindlimb motoneurones in the cat. Exp Brain Res 24:37-55.

Hultborn H, Udo M (1972) Convergence in the reciprocal Ia inhibitory pathway of excitation from descending pathways and inhibition from motor axon collaterals. Acta Physiol Scand 84:95-108.

Ito M, Hongo T, Yoshida Y, Okada Y, Obata K (1964) Antidromic and trans-synaptic activation of Deiters' neurones induced from the spinal cord. Jpn J Physiol 14:638-658.

Jankowska E, Hammar I, Slawinska U, Maleszak K, Edgley SA (2003) Neuronal basis of crossed actions from the reticular formation upon feline hindlimb motoneurons. J Neurosci 23:1867-1878.

Kasper J, Schor RH, Wilson VJ (1988) Response of vestibular neurons to head rotations in vertical planes. II. Response to neck stimulation and vestibular-neck interaction. J Neurophysiol 60:1765-1778.

Kasper J, Schor RH, Wilson VJ (1989) Neck-vestibular interaction in the vestibular nuclei. A dynamic, two-dimensional study. Acta Otolaryngol Suppl 468:137-139.

Kozhanov VM, Shapovalov AI (1977) Synaptic organization of supraspinal control of the propriospinal neurons of the ventral horn of the cat and monkey spinal cord [in Russian]. Neirofiziologiia 9:177-184.

Kuze B, Matsuyama K, Matsui T, Miyata H, Mori S (1999) Segment-specific branching patterns of single vestibulospinal tract axons arising from the lateral vestibular nucleus in the cat: a PHA-L tracing study. J Comp Neurol 414:80-96.

Lund S, Pompeiano O (1965) Descending pathways with monosynaptic action on motoneurones. Experientia 21:602-603.

Lund S, Pompeiano O (1968) Monosynaptic excitation of $\alpha$-motoneurones from supraspinal structures in the cat. Acta Physiol Scand 73:1-21.

Lundberg A (1979) Multisensory control of spinal reflex pathways. Prog Brain Res 50:11-28.

Maeda M, Maunz RA, Wilson VJ (1975) Labyrinthine influence on cat forelimb motoneurons. Exp Brain Res 22:69-86.

Peterson BW, Felpel LP (1971) Excitation and inhibition of reticulospinal neurons by vestibular, cortical, and cutaneous stimulation. Brain Res 27:373-376.

Rovainen CM (1979) Electrophysiology of vestibulospinal and vestibuloreticulospinal systems in lampreys. J Neurophysiol 42:745-766.

Shapovalov AI (1969) Posttetanic potentiation of monosynaptic and disynaptic actions from supraspinal structures on lumbar motoneurons. J Neurophysiol 32:948-959.

Skinner RD, Remmel RS (1978) Monosynaptic inputs to lumbar interneurons from the lateral vestibular tract and the medial longitudinal fasciculus. Neurosci Lett 10:259-264.

Takakusaki K, Kohyama J, Matsuyama K, Mori S (2001) Medullary reticulospinal tract mediating the generalized motor inhibition in cats: parallel inhibitory mechanisms acting on motoneurons and on interneuronal transmission in reflex pathways. Neuroscience 103:511-527.

ten Bruggencate G, Burke R, Lundberg A, Udo M (1969) Interaction between the vestibulospinal tract, contralateral flexor reflex afferents, and la afferents. Brain Res 14:529-532.

Wilson VJ, Peterson BW (1978) Peripheral and central substrates of vestibulospinal reflexes. Physiol Rev 58:80-105.

Wilson VJ, Schor RH (1999) The neural substrate of the vestibulocollic reflex. What needs to be learned. Exp Brain Res 129:483-493.

Wilson VJ, Yoshida M (1969) Comparison of effects of stimulation of Deiters' nucleus and medial longitudinal fasciculus on neck, forelimb, and hindlimb motoneurons. J Neurophysiol 32:743-758.

Wilson VJ, Yamagata Y, Yates BJ, Schor RH, Nonaka S (1990) Response of vestibular neurons to head rotations in vertical planes. III. Response of vestibulocollic neurons to vestibular and neck stimulation. J Neurophysiol 64:1695-1703. 